\section{Les nouvelles de ce numéro}

ont été préparées par:

\section{Eduardo Anglés-Cano ${ }^{(1)}$}

Pascale Briand

Elisabeth Bursaux

Erick Denamur ${ }^{(2)}$

Jean-Claude Dreyfus

Jean-Pierre Grünfeld

Axel Kahn

Vincent Lotteau

Marc Peschanski

Chantal

Rabourdin-Combe ${ }^{(3)}$

Christian de

Rouffignac $^{(4)}$

\section{SOMMAIRE DES NOUVELLES BRÈVES}

Facteurs de croissance et glomérulonéphrites (p. 219).

Encore l'oncogène Bcl-2 (p. 219).

Localisation chromosomique et clonage du gène de la maladie de Krabbe (p. 221)

La production de NO par l'endothélium vasculaire est-elle diminuée dans le diabète sucré insulinodépendant (p. 223).

La faible réponse anticoagulante à la protéine $C$ activée: un mécanisme nouveau de thrombose familiale (p. 231).

Interféron $\gamma$ et tuberculose (p. 231).

Confirmation du rôle de la graisse brune dans la régulation du poids corporel (p. 232).

Interleukine 1, apoptose et sénescence (p. 232).
Le partenaire commun des récepteurs des interleukines 2,4 et 7 (p. 233).

Le précurseur des lymphocytes Th1 et Th2 exprime le gène de l'interleukine 4 (p. 233).

Le ligand de Fas est un membre de la famille du facteur nécrosant des tumeurs (p.234).

Le CD46 est le récepteur du virus de la rougeole (p. 234).

Influence du polymorphisme sur la spécificité des transporteurs de peptides TAP (p. 235).

Les mâles sont pressés (p. 235).

Raf-1, la cible de l'antagonisme entre les signaux relayés par les tyrosine kinases et I'AMP cyclique (p. 235).

\title{
Empreinte génomique et méthylation
}

(1) Inserm U. 143, hôpital de Bicêtre, 78, ave nue du Général-Leclerc, 94275 Le Kremlin Bicêtre Cedex. France.

(2) Inserm U. 120, hôpital Robert-Debré, 48, bd Sérurier, 75935 Paris Cedex 19, France. (3) Laboratoire de biologie moléculaire et cellulaire, UMR CNRS/ENS 49, École Normale Supérieure, 46, allée d'Italie, 69364 Lyon Cedex 07, France.

(4) Département de biologie cellulaire et moléculaire, Centre d'Études de Saclay, 91191 Gifsur-Yvette Cedex, France.

chromosome 7 est d'origine unique-

On parle d'empreinte génomique ou parentale lorsqu'un gène est exprimé de façon différente selon le parent dont il est hérité $\left(\mathrm{m} / \mathrm{s} n^{\circ} 1\right.$, vol. 6, p. 57; $n^{\circ} 3$, vol. 7 , p. 247; $n^{\circ} 3$, vol. 9, p. 333). Il en existe quelques exemples en pathologie humaine; mais nos connaissances viennent surtout de trois gènes de la souris: le gène $H 19$, qui apparemment code pour un ARN mais non pour une protéine, et deux gènes physiologiquement apparentés, codant pour le facteur de croissance IGF-2 et son récepteur IGF-2R. Les gènes $H 19$ et Igf2 sont situés sur le chromosome 7 de la souris, séparés par moins de $100 \mathrm{~kb}$, et sont «imprimés » en sens inverse: H19 est exprimé uniquement sous la direction de l'allèle maternel, Ig/2 de l'allèle paternel; des embryons disomiques, dont le ment paternelle ou maternelle, ne sont pas viables. Igf2r est sur le chromosome 17 et est imprimé à l'inverse de $\operatorname{Ig} 2$ : seul l'allèle maternel est actif. Pour interpréter ces phénomènes, la première idée qui vient est celle d'une différence de méthylation. On en a certes trouvé mais qui ne sont pas aisément explicables [1], et, par ailleurs, certaines méthylations sont tardives et pourraient être la conséquence et non la cause de la différenciation. Un progrès considérable récent est dû à des chercheurs du MIT (Cambridge, MA, USA). Par une technique de ciblage génique faisant appel à la recombinaison homologue [2], ils ont inactivé l'ADN méthyltransférase, d'abord in vitro dans des cellules ES, puis, in vivo, après intro- 
duction de ces cellules dans des souris. Le niveau général de méthylation chez les déficients homozygotes, tant en culture qu' in vivo, tombait au tiers de celui des témoins. Les embryons ainsi traités ne sont pas viables et meurent au milieu de la gestation. Dans un second travail [3], la même équipe, utilisant des embryons âgés de 10,5 jours, a fait porter son analyse sur les trois gènes "imprimés". Le résultat fondamental est la disparition de toute impression parentale spécifique. Le gène paternel $H 19$, normalement silencieux, devient exprimé, de sorte que son taux d'expression double par rapport aux témoins; des méthodes de croisement permettant d'identifier les allèles confirment la dérépression de l'allèle paternel, qui, consécutive à une déméthylation, est conforme à ce que l'on attendait. L'interprétation des résultats portant sur les deux autres gènes est, en revanche, plus difficile. Igf2 et, dans une moindre mesure, $\lg f 2 r$, sont réprimés chez les souris homozygotes pour le déficit en méthyltransférase. Un niveau normal de méthylation générale semble donc indispensable au comportement correct des gènes imprimés. Les expériences de Li et al. montrent donc, de façon irréfutable, l'importance vitale des méthylations; elles suggèrent l'importance du maintien à l'état normal des gènes soumis à impression génomique, mais, elles suggèrent aussi que le mécanisme de leur activationrépression comporte encore nombre d'inconnues.

A partir de là, puisqu'un modèle simple de méthylation-répression ne convient pas, on est conduit à évoquer des modèles réclamant quelques acrobaties conceptuelles $[1,4]$. En ce qui concerne le gène Igf-2r: il possède deux îlots $\mathrm{CpG}$ distincts; l'un, dans un intron $27 \mathrm{~kb}$ en aval du promoteur, est méthylé sur l'allèle maternel ; l'autre, situé sur le promoteur, est méthylé sur l'allèle paternel qui est inactivé. L'îlot $\mathrm{CpG}$ intronique, méthylé sur la copie active du gène, agit peut-être comme silencer de l'allèle paternel lorsqu'il n'est pas méthylé, en liant des molécules répresseur, par exemple. Il serait donc mis en service chez les homozygotes déficients en

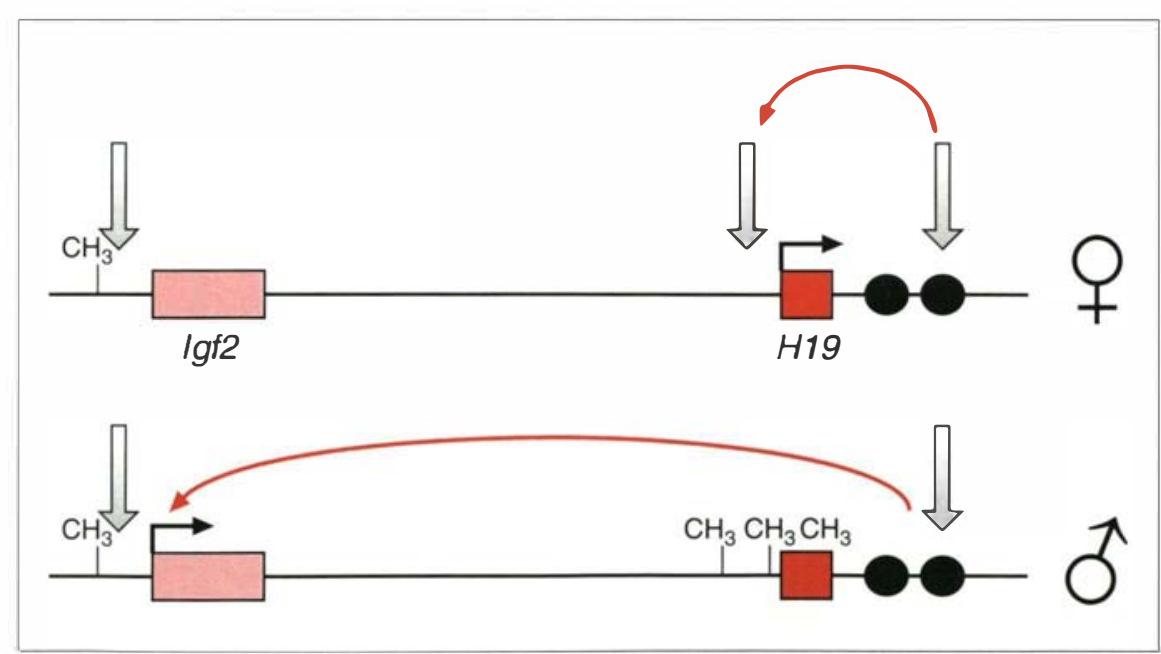

Figure 1. Le modèle de compétition pour l'enhancer, une explication plausible de l'empreinte inversée des gènes H19 et IGF2. Les gènes H19 et IGF2 sont représentés par des rectangles, la transcription allélique par une flèche horizontale, les deux enhancers de $\mathrm{H} 19$ par les cercles pleins. Les sites de méthylation spécifiques de l'allèle sont indiqués par le symbole $\mathrm{CH} 3$; les régions hypersensibles décelées sur la chromatine sont indiquées par les flèches doubles verticales. Les flèches rouges partant des enhancers indiquent leur engagement, avec le gène $\mathrm{H} 19$ pour l'allèle paternel, avec le gène IGF2 pour l'allèle maternel. (D'après [4]).

$\mathrm{m} / \mathrm{s} n^{\circ} 2$ vol. 10 , frurier 94 méthyltransférase, ce qui rendrait compte d'une certaine répression d'Igf $2 r$.

Pour les gènes Igf2 et H19, les choses sont encore plus complexes. La méthylation et la condensation de la chromatine du promoteur paternel inactif de $H 19$ pourraient être les éléments du contrôle des deux gènes si l'empreinte génomique de $H 19$ et $I g f 2$ était liée de façon quasiment mécanique: on pourrait imaginer que les promoteurs des deux gènes soient en compétition pour un même enhancer en aval de H19. Cet enhancer n'activerait l'expression d'Igf2 que si le gène $H 19$ était méthylé et inactif, comme cela se passe sur le chromosome paternel (figure 1). Il faut noter que la méthylation de l'ADN et la structure chromatinienne des deux gènes parentaux Igf2 sont peu différentes; le gène Igf2 maternel inactif retient une configuration chromatinienne ouverte malgré le bas niveau de transcription. Chez les homozygotes déficients en méthyltransférase, l'enhancer de chaque allèle interagirait avec le promoteur de $H 19$, et ne serait ainsi plus disponible pour activer le gène $\operatorname{Igf} 2$.

J.C.D. E.B.

1. Surani MA. Silence of the genes. Nature 1993 ; 366 : 302-3.

2. Ij E, Bestor TH, Jaenish R. Targeted mutation of the DNA methyltransferase gene results in embryonic lethality. Cell 1992;69: 915-26.

3. Li E, Beard C, Jaenish R. Role for DNA methylation in genomic imprinting. Nature 1993 ; 366 : 362-5

4. Bartolomei M, Webber AL, Brunkow ME, Tilghman SM. Epigenetic mechanisms underlying the imprinting of the mouse $\mathrm{H} 19$ gene. Genes Dev 1993; 7: 1663-73.
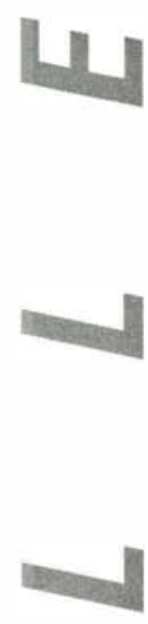

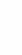

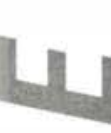

